ABS67: Developing and validating a questionnaire to evaluate the impact of patient-centred asthma training on GPs and practice nurses

Jennifer Cleland, Kathryn Mearns, Kym Baker

Department of General Practice and Primary Care, Foresterhill Health Centre, Westburn Road Aberdeen, AB25 2AY, United Kingdom

Introduction: UK evidence-based guidelines recommend helping those with asthma to control their symptoms through education and individualised, written action plans. Training professionals to help a range of patients with individual disease characteristics, personal attributes and lifestyles may be an important factor in asthma management. Training in patientcentred, communication has proven benefits at the level of randomised controlled trials [1,2]. However, we do not know in what way these interventions are effective. Psychological theories of behaviour change such as the theory of planned behaviour (TPB) [3] can be helpful in determining exactly why an intervention was effective, or not. This can provide information on training variants and their possible effects on outcome. Aims and objectives: To develop and validate a questionnaire using the TPB to explore GP and practice nurses' beliefs and attitudes towards training in patient-centred asthma management. This training is modified from a programme successfully implemented with American office based paediatricians [5]. Methods: We constructed a TPB questionnaire using published guidelines [4] and with reference to the goals of the training programme [5]. The resultant questionnaire was validated through a webbased survey of GPs and practice nurses. Data was analysed using multiple regression. Results and conclusions: The process of constructing and validating the TPB questionnaire will be described, and its use discussed in relation to primary care respiratory research and training.

Conflict of interest and funding None.

References

[1] Razav, et a.l. Guropean 1993; $29 \%: 1053-63$

[2] Fallowfield, et al. The Lancet 200<;539:650-6.

[3] Ajzen I. Org Beh Human Dec Proc 1991;50:179-211.

[4] Francis, et al. Constructing questionnaires based on the theory of planned behaviour: a manual for Health Services researchers. University of Newcastle: Centre for Health Services Research; 2004.

[5] Clark, et al. Paediatrics 1998;101:831-6.

doi:10.1016/j.pcrj.2006.04.161

\section{ABS68: Use of a call centre to conduct clinical research}

Gaylor Hoskins, Ron Neville, Peter Donnan, Colin McCowan, Bridget Shepherd

University of Dundee, Mackenzie Building, Kirsty Semple Way, Dundee, DD2 4BF, United Kingdom

Introduction: Call centres are increasingly utilised to provide health-care services and advice. Their use in research to monitor and track individuals with long-term health conditions such as asthma without subjecting them to regular clinic visits has yet to be validated. Aim: As part of an asthma health outcomes study a call centre conducted monthly interviews with patients over one year. The relationship between the research team and the call centre, as well as data quality, was reviewed. Method: 344 patients from 31 randomly selected UK practices were called on a monthly basis. The interview included 5 questions on asthma symptoms and reliever inhaler use (generic EQ-5D), 11 on Health Service resource use due to asthma, one question on personal costs, and a self reported Peak Flow reading. Responses were recorded in Access and transferred to the research unit for analysis. At the end of the study patients were asked to give their views on the process. The data was analysed for successful interview completion and patient response. Results: Six patients were excluded prior to the interview process as they did not fit the inclusion criteria; a further 30 did not complete any monthly interviews. 2838 interviews from 308 patients were available for analysis. Seventy (23\%) patients completed 12 or more monthly interviews and $200(65 \%)$ between 6 and 11, but $28(9 \%)$ had 5 or less completed interviews. Patients expressed mixed views about the timing, commitment, and standard of service offered by the call centre. Conclusion: Call centres can be used in clinical research if safeguards are in place. Their ability to handle large volumes of interviews needs to be offset by the provision of strict trial protocols; an effective trial coordinator, and frequent monitoring of the call centre process.

\section{Conflict of interest and funding}

Conflict of interest none.

This project was sponsored and funded by a grant from GlaxoSmithKline (UK).

doi:10.1016/j.pcrj.2006.04.162

ABS69: Using information effectively to develop a local enhanced service for COPD

Sally Hull

Institute of Health Sciences Education, Barts and the London, 2, Newark Street, Whitechapel, London, E1 2AT, United Kingdom

Introduction: In the United Kingdom $5 \%$ of mortality can be attributed to Chronic Obstructive rulmonaly bisease (COPD). Exacerbations of COPD. exacerbition; of CUlD account for $10 \%$ of acute medicil ac n ssirns with rates in east London sionificanly isher than elsewhere in the UK.

(ith an improved evildence base for interventions which can reduce syinnto is = nc prevent exacerbations, attention has tured tc the systematic management of COPD across the boundar, of primary and secondary care. The nGMS contract pro/ides incentives to develop registers, improve diagnosis using spirometry and record chronic care details. integrating these measures with early hospital discharge schemes, and the effective implementation of guidelines remains a challenge. Aim: To discuss developing a practice based local enhanced service to build practice capacity in COPD. as practice based commissioning develops, the enhanced service will form a stepping stone towards a service model which crosses boundaries between practices and between hospital and primary care. Method: Core elements necessary for chronic disease management in COPD are described. These include:

1. The role of a clinical effectiveness unit in providing guidelines, facilitation, data entry templates and audit.

2. A local enhanced service for COPD.

3. A community based spirometry service.

4. A dedicated locality clinical lead.

Findings: We will demonstrate the importance for primary care organisations which utilise data analysis techniques which go beyond the quality and outcome framework. This will be demonstrated in relation to:

\section{Admission data by practice}

Linkage to hospital PAS systems for spirometry results Data collection and audit for the COPD enhanced service.

Discussion: The limited evidence base for some of the system interventions will be discussed. Some of the barriers to change will be identified, particularly those which affect interventions of proven clinical benefit.

\section{Conflict of interest and funding}

None.

doi:10.1016/j.pcrj.2006.04.163 